\section{Enteropathy-associated T cell lymphoma as a complication of silent celiac disease}

\author{
Margarida Dantas Brito, ${ }^{1}$ \\ Ângelo Martins, ${ }^{1}$ Rui Henrique, ${ }^{2}$ \\ José Mariz ${ }^{1}$ \\ 1Department of Hematology, \\ 2Department of Pathology, Portuguese \\ Institute of Oncology of Porto, Portugal
}

\begin{abstract}
Celiac disease is an autoimmune disorder in which a genetic predisposition and the ingestion of wheat gluten triggers a deleterious immune response. This response is complex and may lead to manifestations other than enteropathyha: hepatitis, dermatitis and neuropathy. There is higher risk for neoplasia. We observed an atypical case, corresponding to a 69-year old female presenting with complicated celiac disease. The patient was referred following the histological examination of an enterectomy specimen, which unexpectedly revealed an enteropathy-associated T cell lymphoma in a background of celiac disease. Patient's previous medical history comprised several abdominal surgical procedures, without other prior symptoms suggestive of celiac disease. Indeed, the patient was obese and no signs of malabsortion were apparent. This case draws our attention to clinically silent celiac disease, which represents a diagnostic challenge. Thus, this should be kept in mind whenever a patient presents with abdominal relapsing complications, otherwise unexplained.
\end{abstract}

\section{Introduction}

Celiac disease $(\mathrm{CeD})$ is a complex disorder of the immune system in which patients have a genetic predisposition and the ingestion of wheat gluten (or related proteins) triggers a deleterious immune response. Tissue glutaminase binds these ingested peptides and presents them to $\mathrm{T}$ cells that lead to $\mathrm{B}$ cell production of autoantibodies. Innate immunity is also involved targeting gliadin and tissue transglutaminase, causing inflammatory response with tissue damage. ${ }^{1,2}$ This immune response is complex and may lead to manifestations other than enterophaty: hepatitis, dermatitis, thyroid disorders and neuropathy. ${ }^{1-4}$ There is also a higher risk for neoplasia. Among these, enteropathy-associated $\mathrm{T}$ cell lymphoma (EATL) is a very rare malignancy (incidence 0.5 to 1 per million). The 2008 World Health
Organization classification of tumors of hematopoietic and lymphoid tissues recognizes two types of EATL, according to morphologic and genetic features. EATL type 1 is more frequently associated with $\mathrm{CeD}$, usually arising as late complication of refractory disease and carrying a dismal prognosis. ${ }^{5,6}$

Several studies shown a trend for increasing incidence of $\mathrm{CeD}$ and up to 20 or $30 \%$ of patients are diagnosed after the age of sixty. Clinical manifestations in elderly patients remain largely uncharacterized. -10 $^{7-10}$

\section{Case Report}

We have recently observed an atypical clinical case, corresponding to a 69-year old Caucasian female presenting with complicated celiac disease and EATL at diagnosis. The patient was referred to the Department of Hematology from the Department of Surgery following the histological examination of a partial enterectomy specimen, which unexpectedly revealed EATL in a background of CeD. Patient's previous medical history comprised obesity and several abdominal surgical procedures. In May 2008, a laparotomy was performed to correct an incisional hernia and few months later she developed bowel obstruction requiring laparotomy. The cause of the obstruction was not found. Gradually the incisional hernia relapsed and in May 2013 a small bowel enterocutaneous fistula became apparent, entailing another laparotomy in which a partial enterectomy was performed. Grossly, the specimen showed a diffuse whitish thickening of the intestinal wall and histological examination revealed typical features of EATL type 1, as well as histological changes suggestive of $\mathrm{CeD}$ in the adjacent mucosa (Figure 1). The tumor immunophenotype was: $\mathrm{CD} 2+$, $\mathrm{CD} 3+$, and $\mathrm{CD} 5+$ (low expression), CD8+, CD30+, CD4-, CD20-, CD15-, CD56-, ALK-. In situ hybridization for EBV RNA (EBER-1) was positive.

At the time of admission in the Department of Hematology (five months after the last surgery), the patient showed minor dehiscence of the abdominal incision with no further complaints. We sought for other clinical complaints that could suggest $\mathrm{CeD}$, but besides the occlusive episode, the patient referred regular bowel function and no systemic complaints. Blood analyses were entirely normal, with no signs of anemia or liver abnormalities. Serologic testing for antigliadin antibodies and transglutaminase antibodies (IgA and IgG) were negative. The deamidated gliadin peptides assay was not performed because it is not available at our institution. Colonoscopy revealed stenosis and ulceration at the enterocolic anastomosis (Figure 2). Bone marrow
Correspondence: Margarida Dantas Brito, Department of Hematology, Portuguese Institute of Oncology of Porto, Rua Dr. António Bernardino de Almeida, 4200 Porto, Portugal.

Tel.: +351.225.084.000 - Fax: +351.225 .084 .001 .

E-mail: ana.margarida.dantas@gmail.com

Key words: lymphoma, celiac disease, elderly.

Contributions: the authors contributed equally.

Conflict of interests: the authors declare no potential conflict of interests.

Received for publication: 25 August 2014. Revision received: 2 November 2014.

Accepted for publication: 13 November 2014.

This work is licensed under a Creative Commons Attribution NonCommercial 3.0 License (CC BYNC 3.0).

(C) Copyright M.Dantas Brito et al., 2014

Licensee PAGEPress, Italy

Hematology Reports 2014; 6:5612

doi:10.4081/hr.2014.5612

biopsy was normal. Staging computed tomography scan was consistent with localized disease and thus the patient was considered stage II according to the Lugano staging system for gastrointestinal lymphomas. ${ }^{11}$ Although staging procedures are not standardized for this lymphoma, video capsule enteroscopy and 18F-fluorodeoxyglucose positron emission tomography are clinical options.

The patient was treated with six cycles of chemotherapy according to the CHOP (cyclophosphamide, doxorubicin, vincristine and prednisolone) protocol and referred for nutritional counseling. Between chemotherapy cycles the patient received hematopoietic growth factor (G-CSF) prophylactic treatment. The minor dehiscence of the abdominal incision showed on several occasions signs of mild infection. She had regular nursing wound care and often needed oral antibiotic prescription, according to the microbiology swabs. The patient never experienced neutropenic fever or other signs of severe infection.

At the end of chemotherapy the patient was in complete remission: both the computed tomography scan and biopsy of enterocolic anastomosis were negative for neoplasia. High-dose chemotherapy and autologous stem cell transplantation was not pursued since this was an elderly patient, prone to complications during this aggressive treatment and had localized disease at diagnosis. Currently she is planned for clinical and endoscopic follow-up. Computed tomography scan can provide further information if relapse is suspected. It has been difficult to alter the nutritional habits of 
this elderly patient, but since the EATL diagnosis the patient is receiving nutritional counseling to follow a gluten-free diet.

\section{Discussion}

In the context of $\mathrm{CeD}$, this patient displayed a very atypical clinical picture, presenting with enterophaty-related lymphoma concomitant with histological changes suggestive of $\mathrm{CeD}$ in
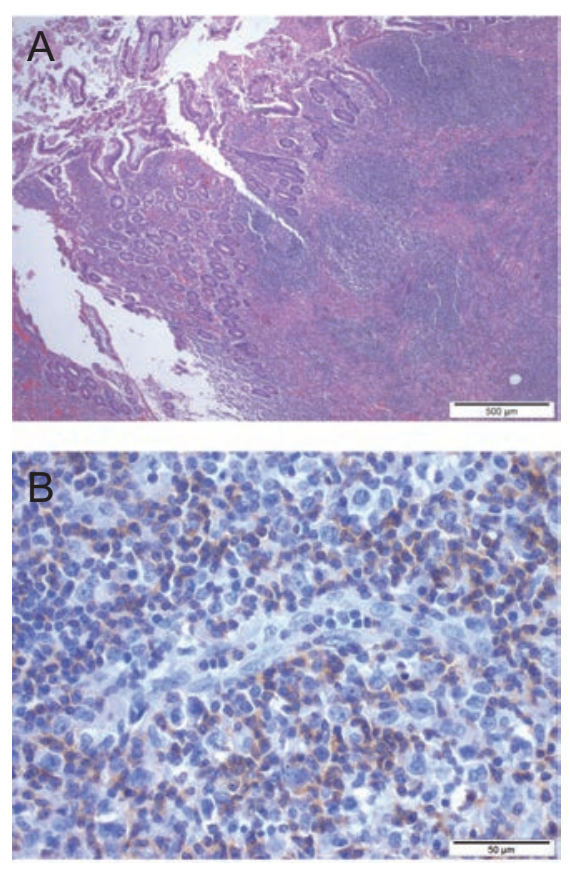

Figure 1. A) Low-power view of the lymphoma, involving the intestinal wall and ulcerating the mucosa (HE staining). B) Immunoreactivity for CD3 in small and intermediate-size neoplastic cells; large cells have lost positivity for CD3.

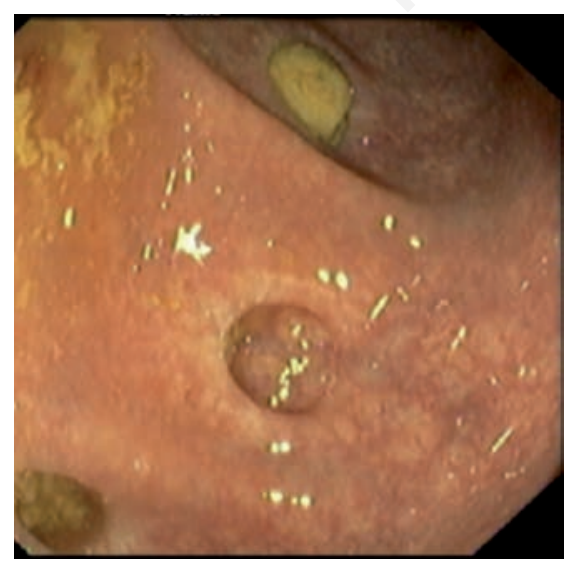

Figure 2. Photography from colonoscopy showing stenosis and ulceration at the enterocolic anastomosis. the adjacent mucosa, without prior symptoms suggestive of CeD. Indeed, the patient is obese and no signs of malabsortion were apparent. Moreover, no antibodies against gliadin were detected. Although specific, its absence does not exclude CeD. ${ }^{2-4}$ One may question if the previous abdominal relapsing complications that required multiple laparotomies across several years were due to silent $\mathrm{CeD} .^{5,6}$

This atypical clinical presentation contributed to diagnosis delay and lack of specific measures that could have contributed to prevent the evolution towards EATL. The patient was exposed to gluten during all her lifetime and it is widely acknowledged that a glutenfree diet effectively prevents the development of EATL in patients with overt CeD. ${ }^{12,13}$ In this clinical case there was no formal indication to institute a gluten-free diet previous to the diagnosis of lymphoma, since the diagnosis of CeD was simultaneous to that of EATL. One may question whether silent $\mathrm{CeD}$ patients would benefit from an earlier institution of gluten-free diet in order to prevent those events.Enteropathy-associated T cell lymphoma is frequently diagnosed after pathologic evaluation of a resected specimen of small bowel due to intestinal obstruction or perforation. Patient's clinical conditions are often a major contraindication to start chemotherapy. Complications such as perforation, infection, fistulae and poor nutritional status related with chronic malabsorption may postpone systemic treatment. This plays a relevant role in defining part of the poor prognosis of this disease. ${ }^{14,15}$ Nutritional support, sometimes with parenteral nutrition, and rigorous control of infectious complications are critical issues in the management of these patients. Fortunately, our patient did not experience any of those problems. Although she had a minor dehiscence of the abdominal incision she was able to tolerate chemotherapy.

This patient received six cycles of chemotherapy according to the CHOP protocol. There is no standard treatment approach for this rare lymphoma. Usually the treatment consists of a combination chemotherapy regimen used for other aggressive $\mathrm{T}$ cell lymphomas. ${ }^{14,15}$ This patient was not a candidate for autologous hematopoietic transplantation, but some studies (based in small number of patients) suggest survival benefit with transplantation in first remission when patients are eligible. ${ }^{16,17}$

Generally EATL is an aggressive lymphoma with high-grade histology and poor prognosis, usually worse than other intestinal lymphomas. The major determinants of prognosis are stage at presentation and clinical status to allow for systemic treatment. Occasional patients with localized disease may be cured but many patients will die from complications of multifocal intestinal perforation or systemic progression. The five-year overall survival rate with anthracycline-based chemotherapy is approximately 10 to 20 percent. ${ }^{14,15}$

\section{Conclusions}

This case draws our attention to clinically silent $\mathrm{CeD}$, which represents a diagnostic challenge. Thus, this should be kept in mind whenever a patient presents with abdominal relapsing complications which are otherwise unexplained.

\section{References}

1. Green PHR, Cellier C. Celiac disease. N Engl J Med 2007;357:1731-43.

2. Catassi C, Kryszak D, Bhatti B, et al. Natural history of celiac disease autoimmunity in a USA cohort followed since 1974. Ann Med 2010;42:530-8.

3. Dieterich W, Ehnis T, Bauer M, et al. Identification of tissue transglutaminase as the autoantigen of celiac disease. Nat Med 1997;3:797-801.

4. D’Angelo S, Mignone F, Deantonio C, et al. Profiling celiac disease antibody repertoire. Clin Immunol 2013;148:99-109.

5. Carbonnel F, Grollet-Bioul L, Brouet JC, et al. Are complicated forms of celiac disease cryptic T-cell lymphomas? Blood 1998; 92:3879-86.

6. Catassi C, Fabiani E, Corrao G, et al. Risk of non-Hodgkin lymphoma in celiac disease (for the Italian Working Group on Coeliac Disease and Non-Hodgkin'sLymphoma). JAMA 2002;287:1413-9.

7. Rashtak S, Murray JA. Celiac disease in the elderly. Gastroenterol Clin North Am 2009;38:433-46

8. Vilppula A, Kaukinen K, Luostarinen L, et al. Increasing prevalence and high incidence of celiac disease in elderly people: a population-based study. BMC Gastroenterol 2009;9:49.

9. Freeman HJ. Adult celiac disease in the elderly. World J Gastroenterol 2008;14: 6911-4.

10. Yoav L, Dan-Avi L, Pfeffer J, et al. Celiac disease diagnosed in the elderly. J Clin Gastroenterol 2008;42:59-61.

11. Rohatiner A, d'Amore F, Coiffier B, et al. Report on a workshop convened to discuss the pathological and staging classifications of gastrointestinal tract lymphoma. Ann Oncol 1994;5:397-400.

12. Silano M, Volta U, Vincenzi AD, et al. Effect of a gluten-free diet on the risk of enteropathy-associated T-cell lymphoma in celiac disease. Collaborating Centers of 
the Italian Registry of the Complications of Coeliac Disease. Dig Dis Sci 2008;53:972-6.

13. Lebwohl B, Granath F, Ekbom A, et al. Mucosal healing and risk for lymphoproliferative malignancy in celiac disease: a population-based cohort study. Ann Intern Med 2013;159:169-75.

14. Delabie J, Holte H, Vose JM, et al. Enteropathy-associated T-cell lymphoma: clinical and histological findings from the international peripheral T-cell lymphoma project. Blood 2011;118:148-55.

15. Gale J, Simmonds PD, Mead GM, et al. Enteropathy-type intestinal T-cell lymphoma: clinical features and treatment of 31 patients in a single center. J Clin Oncol 2000;18:795-803.

16. Bishton MJ, Haynes AP. Combination chemotherapy followed by autologous stem cell transplant for enteropathy-associated T cell lymphoma. Br J Haematol 2007;136:111-3.

17. Sieniawski M, Angamuthu N, Boyd K, et al. Evaluation of enteropathy-associated Tcell lymphoma comparing standard therapies with a novel regimen including autologous stem cell transplantation. Blood 2010;115:3664-70. 